\title{
24-h Activity Rhythms and Health in Older Adults
}

\author{
Maud de Feijter ${ }^{1} \cdot$ Thom S. Lysen $^{1} \cdot$ Annemarie I. Luik ${ }^{1}$
}

Published online: 10 March 2020

(C) The Author(s) 2020

\begin{abstract}
Purpose of Review Circadian rhythms, including 24-h activity rhythms, change with age. Disturbances in these 24-h activity rhythms at older age have also been implied in various diseases. This review evaluates recent findings on $24-\mathrm{h}$ activity rhythms and disease in older adults.

Recent Findings Growing evidence supports that 24-h activity rhythm disturbances at older age are related to the presence and/or progression of disease. Longitudinal and genetic work even suggests a potential causal contribution of disturbed 24-h activity rhythms to disease development. Interventional studies targeting circadian and 24-h activity rhythms demonstrate that 24-h rhythmicity can be improved, but the effect of improving 24-h rhythmicity on disease risk or progression remains to be shown. Summary Increasing evidence suggests that 24-h activity rhythms are involved in age-related diseases. Further studies are needed to assess causality, underlying mechanisms, and the effects of treating disturbed 24-h activity rhythms on age-related disease.
\end{abstract}

Keywords Circadian rhythm $\cdot$ Rest-activity rhythm $\cdot$ Aging $\cdot$ Age-related disease $\cdot$ Actigraphy $\cdot$ Accelerometry

\section{Introduction}

The circadian rhythm is integral to physiological processes throughout the body [1]. These approximately 24-h rhythms are regulated by the master clock located in the brain's suprachiasmatic nucleus (SCN) [2], and are shaped using endogenous and exogenous cues. Together, this ensures that our physiological functioning can be optimized and adapted to changing environmental conditions and social demands [3].

Circadian rhythms can be observed in a range of physiological and behavioral processes throughout the body, for example, fluctuations in clock gene expression, hormone levels, body temperature, and cognitive processes [1]. Although many of these fluctuations are valid and precise markers of the circadian rhythm, they are often less feasible to study when the circadian rhythm needs to be assessed over longer periods of time or in large populations. An accessible, affordable, and unobtrusive alternative to measure 24-h rhythmicity is actigraphy, also known as accelerometry. Actigraphy can

This article is part of the Topical Collection on Sleep and Aging

Annemarie I. Luik

a.luik@erasmusmc.nl

1 Department of Epidemiology, Erasmus MC University Medical Center, Rotterdam, The Netherlands measure activity continuously over multiple days, weeks, or even months. Naturally, activity is under voluntary control and may therefore misrepresent some of the underlying endogenous rhythms. Yet, measuring 24 -h activity rhythms with actigraphy has been demonstrated to be a valid method to estimate circadian rhythmicity, in both patients and healthy adults $[1,4]$. With ever increasing recording quality, better storage capacities, longer recording lengths, and the availability of open source algorithms, actigraphy has become a mainstay for studying circadian rhythms in research and clinical practice $[5 \cdot, 6]$.

Circadian rhythms, and associated 24-h activity rhythms, are altered with increasing age $[1,7 \cdot]$. Older age is also accompanied by an increase in the prevalence of noncommunicable diseases [8]. It has been hypothesized that changes in 24-h activity rhythms might indicate poor health or even pose a risk factor for poor health, not the least because fragmented 24-h activity rhythms have been associated with an increased risk of mortality [9]. As modern $24 / 7$ society puts a widespread strain on our rhythms, for example, through artificial lighting and shift work, it is crucial to better understand the role of 24-h activity rhythm disturbance in the development of age-related disease. In this review, we will briefly discuss the measurement of 24-h activity rhythms, give a short overview of age-related changes in 24-h activity rhythms, and discuss recent findings around 24-h activity 
rhythms and some of the most common diseases in older adults.

\section{Measuring the 24-h Activity Rhythm}

A range of scientific grade actigraphs, typically worn around the wrist, has become available over the past decades. Starting with relatively simple devices measuring activity on one axis, nowadays, almost all actigraphs measure movement on three axes and are equipped with additional sensors measuring temperature, light, and/or heart rate. Typically, only the activity data has been used to estimate the 24-h rhythm. Multiple commercial wearables that measure activity have also become available; their value in assessing 24-h activity rhythms remains to be determined [10].

Several methods have been developed to derive 24-h rhythmicity estimates from actigraphy $[4,11 \bullet \bullet, 12 \bullet \bullet]$; the most used methods are based on adapted cosinor models [4, 11••] and non-parametric models [12••]. From these models, a range of 24-h activity rhythm estimates is calculated, which are correlated to some extent [13]. Both methods come with their own set of advantages and disadvantages. For example, nonparametric measures have been suggested to better reflect 24-h rhythms in elderly persons, because rhythms are generally less cosine shaped in older adults [12••]. In contrast, cosinor measures seem to be associated more consistently with outcomes such as cognitive functioning [14]. A description of the most commonly used cosinor and non-parametric estimates can be found in Table 1.

\section{4-h Activity Rhythms and Aging}

Old age is accompanied by multiple changes in the 24-h activity rhythm, including a well-described phase advance [13].
In recent work, a lower amplitude, lower mesor, earlier acrophase, and more fragmented rhythm have been described in older adults $[6,9,15 \bullet, 16,17]$. Daytime activity levels are also lower at old age [18], but nighttime activity levels do not necessarily change in old age [19]. The stability of the 24-h activity rhythm seems to remain similar across ages [16], and has even been suggested to be higher in old age [20]. A recent study in 91,105 individuals suggested that age was not associated with relative amplitude [21•], but this study only included persons aged 73 or younger. This fits previous suggestions that 24-h activity rhythm disturbances are most pronounced in those aged 80 years or older $[9,16]$.

Older and more recent studies thus both demonstrate that middle-aged and elderly persons have "dampened" and less robust 24-h activity rhythms [6], similar to alterations seen in endogenous measures of the circadian rhythm at older age [1]. It is largely unknown to what extent these changes may be attributed to the aging process per se. They could also be caused by environmental changes that accompany older age, such as retirement, less physical activity, or the emergence of age-related diseases. Probably, a combination of endogenous and exogenous factors plays a role in age-related changes in the 24-h activity rhythm [1].

\section{4-h Activity Rhythms and Neurodegenerative Disease}

Over the last 3 years, neurodegenerative disease has been the most studied disease in relation to 24-h activity rhythms. Indeed, circadian disturbances, including disrupted 24-h activity rhythms, are common in neurodegenerative disease [5•, $22,23]$. These disturbances are potentially attributable to neurodegenerative processes directly affecting circadian regulatory circuits $[2,24]$, or indirectly through behavioral symptoms impairing daily functioning and inadequate exposure to
Table 1 Commonly used cosinor and non-parametric $24-\mathrm{h}$ activity rhythm estimates $[4,11 \bullet \bullet, 12 \bullet \cdot]$

\begin{tabular}{ll}
\hline Variable & Explanation \\
\hline Cosinor & \\
Acrophase & Timing of maximum activity (clock time) \\
Amplitude & Difference between maximum and minimum value of activity (score) \\
Mesor & Average activity (counts/min) \\
Period & Time interval over which cycles repeat (hours) \\
Pseudo-F & Fit of activity data to the cosine function, indicating rhythm "robustness" (score) \\
Non-parametric & \\
Interdaily variability & Fragmentation of the rhythm relative to its 24-h amplitude (score) \\
Intradaily stability & Stability of activity profiles over days (score) \\
Relative amplitude & Normalized difference between most active 10 h and least active 5 h (score) \\
M10 onset & Onset of most active 10-h period (clock time) \\
L5 onset & Onset of least active 5-h period (clock time) \\
\hline
\end{tabular}


exogenous cues. Vice versa, disturbed circadian rhythms have also been hypothesized to contribute to neurodegenerative processes $\left[5^{\bullet}\right]$. In the next paragraphs, we will focus on recent findings on the role of 24-h activity rhythm disturbances in Alzheimer's disease and other dementias, and Parkinson's disease.

\section{Alzheimer's Disease and Dementia}

Dementia, of which Alzheimer's disease is the most common subtype, is characterized by progressive cognitive decline and impairment in activities of daily living [25]. Disruptions of 24-h activity rhythms in these patients were first recorded over two decades ago [26], and have been reviewed recently [5•, $14,27]$. These disruptions predominantly include fragmentation and a reduced amplitude of the 24-h activity rhythm, and behaviors such as "sun-downing" [27] and frequent daytime napping [28]. Recent cross-sectional studies report a lower amplitude [28-30], a lower stability [29, 31], and more fragmentation [29] in patients with dementia. More fragmented 24-h activity rhythms were also found in persons with earlyonset dementia [32]. Together, these disturbances substantially impair quality of life of patients and caregivers $[33 \cdot, 34]$ and are thought to be an important determinant for the institutionalization of patients [27].

Research increasingly focuses on the pre-diagnostic phase of dementia to investigate the potential etiological or predictive role that 24-h activity rhythm disturbances may have in dementia. Two recent studies investigated persons with potential prodromal symptoms of dementia, but no evidence was found for an association of phase with subjective cognitive complaints [35] or of amplitude with mild cognitive impairment [30]. This was even though the latter was found to be disturbed in those with Alzheimer's disease [30]. In contrast, some earlier studies have reported a phase advance in persons with mild cognitive impairment compared with healthy controls [36, 37] and a higher fragmentation and lower mesor in those with preclinical AD [16]. Data from prospective cohorts provide some further insight into the temporality of the association of 24-h activity rhythms with dementia. An advanced acrophase was associated with an increased risk of cognitive decline in elderly men [15•], whereas in elderly women, a phase delay and lower robustness of the rhythm were associated with an increased risk of dementia and mild cognitive impairment [38]. A higher fragmentation was also related with a decline in cognition measured over the prior 12 years [39॰].

Associations of 24-h activity rhythms with biomarkers of neurodegeneration in non-demented individuals have been investigated to shed further light on the link between 24-h activity rhythms and dementia. First, fragmentation was most strongly related to a cerebrospinal fluid biomarker profile indicative of Alzheimer's disease, when compared with other disturbances [16]. Additionally, fragmentation was related to temporal lobe atrophy in cognitively unimpaired persons [29] and to loss of gray matter in parietal regions specific to early accumulation of Alzheimer's pathology [39•]. Further research remains needed to determine whether 24-h activity rhythm estimates could also serve as a valid biomarker for dementia.

\section{Parkinson's Disease}

In Parkinson's disease, which has a notable association with REM sleep behavior disorder [5•], 24-h activity rhythm disturbances have been hypothesized to occur early in the disease process and to potentially contribute to various symptoms and pathological processes specific to Parkinson's disease [40]. Patients have a higher fragmentation, lower stability, lower amplitude, and lower mesor than healthy controls [40-42]. A low stability is also associated with poorer cognitive performance in Parkinson's disease [42]. It is unclear to what extent the 24-h activity rhythm estimates are affected by impaired motor functioning associated with the Parkinson's disease diagnosis or dopaminergic treatments for Parkinson's disease [5•].

The longitudinal relation of 24-h activity rhythms with incident Parkinson's disease has received limited attention so far, but a recent study with 11 years of follow-up showed that daytime actigraphy-estimated inactivity, indicative of "napping," was associated with increased risk of Parkinson's disease [43]. Longitudinal studies assessing 24-h activity rhythms in relation to Parkinson's disease in particular, and neurodegenerative disease more broadly, are therefore highly needed.

\section{4-h Activity Rhythms and Late-Life Psychiatric Disease}

Disturbances in 24-h activity rhythms are related to a range of psychiatric disorders such as depression, anxiety, psychosis, and schizophrenia [44•,45•], of which some are also common in old age. Depression is of specific interest in the context of this review as a second peak in the prevalence of depression starts around the age of 60 years. Depression, characterized by a depressed mood or a loss of pleasure as a key symptom, and additionally symptoms such as weight change, changes in sleep, psychomotor agitation/retardation, fatigue, worthlessness, cognitive complaints, or suicidality [46], has a major impact on global health [47].

\section{Depression}

Patients diagnosed with major depressive disorder have a tendency to eveningness, delayed 24-h activity rhythms, a dampened amplitude, and a lower mesor [17, 48•, 49-51]. Disturbed 24-h activity rhythms are also related with the severity of depressive symptoms, even when symptoms are of a 
subclinical level, which is common in elderly persons. A cross-sectional population-based study of middle-aged and elderly persons found an association of a lower stability and higher fragmentation of the 24-h activity rhythm with more depressive symptoms [20,52]. A preference for eveningness and a phase delay were also associated with severity of depressive symptoms [53, 54]. Potentially, the association of 24-h activity rhythms and depressive symptoms differs between men and women; in one study, associations of disturbed 24-h activity rhythms with depressive symptoms were found in men but not in women [55].

Increasing evidence supports that disturbances in the 24-h activity rhythm not only are apparent during the depressive episode, but might also precede depressive episodes or may persist afterwards [49]. A recent UK Biobank study suggested a lower relative amplitude in those with a retrospectively determined lifetime incidence of major depressive disorder, bipolar disorder, and mood instability [21•]. A longitudinal study in elderly men reported that both a late acrophase alone and the combination of an early acrophase with a dampened 24-h activity rhythm amplitude were associated with a faster increase in depressive symptoms over time [56]. Additionally, a genome-wide association study (GWAS) including 71,500 participants reported a possible association between genetic risk of a low relative amplitude and mood disorders [45•].

It remains unclear to what extent associations between disturbances of 24-h activity rhythms and mental health are due to medication use [44•, 57]. There is evidence that these associations are independent of medication use [56], but we also know that some 24-h activity rhythm disturbances are related to medication use. For example, eveningness and phase delay potentially hamper the efficacy of antidepressants $[58,59 \cdot]$, and ketamine might improve 24-h activity rhythms, independent of its effect on mood [60].

\section{Other Psychiatric Disorders in Late Life}

Bipolar disorders, in which the depressive episodes are accompanied by manic episodes, have also been related to disturbances in the 24-h activity rhythm. Associations seem to be state-dependent, with depressive episodes being accompanied by a phase delay and manic episodes being accompanied by a phase advance [61, 62]. However, the disturbances in the 24-h activity rhythms might not only be a state marker as the phase advance of 24-h activity rhythms and lower mesor may persist after successful treatment of bipolar disorder [44].

For anxiety, relatively common at older age, it was shown that more fragmented 24-h activity rhythms were associated with higher odds of having an anxiety disorder [52], and that lower activity levels and a lower mesor were associated with current anxiety [48]. Additionally, disrupted 24-h activity rhythms have also been linked to more suicidal behaviors $[63,64]$, but the causality of this association remains to be determined. Although actigraphy has been used in patients with schizophrenia, 24-h rhythmicity has not often been assessed [65]. Only one study assessed 24-h activity rhythms and did not find an association of 24-h rhythm estimates with positive or negative symptoms of schizophrenia [66].

\section{4-h Activity Rhythms and Other Age-Related Diseases}

Aging is accompanied by an increase in other noncommunicable diseases such as type 2 diabetes, cardiovascular disease, and cancer [8], which have also been suggested to involve circadian and 24-h activity rhythm disturbances [1, 50].

\section{Cardio-Metabolic Disease}

Cross-sectional studies reported prolonged napping [67], a lower amplitude [68], less stable [20, 68, 69], and more fragmented [20] 24-h activity rhythms in middle-aged and elderly persons with a higher body mass index, a well-known risk factor for cardio-metabolic disease. Additionally, less stable 24-h activity rhythms were associated with increased odds of metabolic syndrome and hypertension in elderly women [69]. Longitudinal work suggests that 24-h activity rhythm disturbances might already be apparent early on. A longitudinal population-based study reported that a lower robustness of the rhythm and a lower amplitude were associated with an increased risk of overall cardiovascular disease, and that a lower mesor was associated with an increased risk of coronary heart disease [70 ${ }^{\bullet}$. It has also been repeatedly shown that shift work is a risk factor for cardiometabolic disease, such as type 2 diabetes, obesity, and coronary artery disease $[50,71,72]$; the 24 -h activity rhythm has however not been assessed in these studies. Most of these findings have been based on observational studies, making it difficult to determine the underlying mechanisms. Experimental studies in humans do however suggest that short-term circadian misalignment already affects biomarkers for metabolic disease, such as systolic blood pressure and preclinical states of diabetes $[73,74]$.

\section{Cancer}

Disturbed 24-h activity rhythms are also seen in those suffering from cancer. A study in palliative cancer care reported that 24-h activity rhythms are more disrupted towards the end of life [75]. Several recent studies also reported an association between 24-h activity rhythm disturbance and cancer-related mortality, most prominently in lung cancer patients, where early mortality risks were up to 4 times higher in patients with disrupted 24-h activity rhythms compared with those with robust rhythms [76•,77]. More disturbed 24-h activity 
rhythms were also associated with a shorter survival time in patients suffering from head and neck cancers [78] and patients receiving palliative cancer care [75]. Cancer treatment also seems to affect the 24-h activity rhythm; a recent longitudinal study showed that several 24-h activity rhythm estimates, including amplitude, worsen with each cycle of chemotherapy in women with breast cancer [79].

\section{Research Agenda}

Collectively, these studies suggest that with older age, 24-h activity rhythms are dampened, more fragmented, and more advanced. The presence of 24-h activity rhythm disturbances is associated with various age-related diseases. Most evidence is available for dementia and depression but these have also been the most studied diseases in relation to 24-h activity rhythms. Associations between the 24-h activity rhythm and disease also differ per 24-h activity rhythm estimate, which creates a complex picture. Only a minority of studies has investigated the association of 24-h activity rhythms and health longitudinally, which limits our knowledge on the temporality of associations between 24-h rhythm disturbances and age-related disease. Although there is some evidence for a possible causal role of 24-h activity rhythms in disease at old age, more definitive evidence needs to be generated with sophisticated analyses methods in prospective cohort studies and intervention studies.

Studies specifically intervening on circadian rhythms that take into account the 24-h activity rhythm remain scarce. A number of studies have reported reduced circadian disruption after bright light therapy in patients with dementia [80], Parkinson's disease [81], depression [82], cardiovascular disease [83], and cancer [84]. However, it is largely unknown to what extent intervening on circadian factors and, subsequently 24-h activity rhythms, improves relevant clinical outcomes such as disease progression or mortality. So far, we do know that interventions focusing on advancing circadian timing, such as early morning bright light therapy, have a positive effect on mood. Bright light therapy decreases depression severity in depressed patients [82], and 8 weeks of dawn-dusk stimulation improved mood and reduced anxiety in elderly persons living in a care home [85]. It remains to be determined if improvement in 24-h activity rhythms is a mediating factor.

Together, we feel that three items are essential to add to the research agenda to improve our understanding of the role of 24-h activity rhythms in health at older age. First, implementation of actigraphy in prospective cohort studies has not only been proven feasible, it is also needed to investigate temporality. It particularly creates a unique opportunity if the 24-h activity rhythm disturbances can be studied before the diagnosis of the disease in population-based cohorts. These studies should ideally include repeated measurements of both disease-related constructs and actigraphy to gain more insight in potentially bi-directional associations. Second, studies have reported successful improvement of circadian rhythms and mental health after interventions focused on the 24-h rhythm, but effects on somatic conditions are largely unclear. Well-controlled intervention trials that integrate actigraphy and have longer follow-up periods will be needed to assess whether treatment of disturbed 24-h activity rhythms can reduce disease burden or even alter disease progression or incidence. This could also provide information for any potential preventive effects of targeting 24-h activity rhythm disturbances. Lastly, as new studies become available with high speed, well-executed meta-analyses will be needed to direct the field. For this aim, a standardized approach using the same estimates and methods between studies will be highly beneficial.

\section{Conclusion}

The 24-h activity rhythm is disturbed in a broad range of age-related diseases. In neurodegenerative disease, psychiatric disease, cardio-metabolic disease, and cancer, patients have more phase shifts, lower amplitudes, more fragmented, and less stable 24 -h activity rhythms. An increasing number of longitudinal studies suggest that these disturbed 24-h activity rhythms may also precede disease, but causality remains to be determined. The need for longitudinal observational studies remains substantial, as well as the need for investigating promising interventions for those diseases where circadian disruption could be involved in disease etiology, symptom maintenance, or impaired quality of life.

\section{Compliance with Ethical Standards}

Conflict of Interest The authors declare that they have no conflict of interest.

Human and Animal Rights and Informed Consent This article does not contain any studies with human or animal subjects performed by any of the authors.

Open Access This article is licensed under a Creative Commons Attribution 4.0 International License, which permits use, sharing, adaptation, distribution and reproduction in any medium or format, as long as you give appropriate credit to the original author(s) and the source, provide a link to the Creative Commons licence, and indicate if changes were made. The images or other third party material in this article are included in the article's Creative Commons licence, unless indicated otherwise in a credit line to the material. If material is not included in the article's Creative Commons licence and your intended use is not permitted by statutory regulation or exceeds the permitted use, you will need to obtain 
permission directly from the copyright holder. To view a copy of this licence, visit http://creativecommons.org/licenses/by/4.0/.

\section{References}

Papers of particular interest, published recently, have been highlighted as:

- Of importance

•• Of major importance

1. Hood S, Amir S. The aging clock: circadian rhythms and later life. J Clin Invest. 2017;127(2):437-46.

2. Swaab DF, Fliers E, Partiman TS. The suprachiasmatic nucleus of the human brain in relation to sex, age and senile dementia. Brain Res. 1985;342(1):37-44.

3. Aschoff J, editor. Exogenous and endogenous components in circadian rhythms. Cold Spring Harb Symp Quant. 1960;25:11-28.

4. Ancoli-Israel S, Cole R, Alessi C, Chambers M, Moorcroft W, Pollak CP. The role of actigraphy in the study of sleep and circadian rhythms. Sleep. 2003;26(3):342-92.

5. Leng Y, Musiek ES, Hu K, Cappuccio FP, Yaffe K. Association between circadian rhythms and neurodegenerative diseases. Lancet Neurol. 2019;18(3):307-18. Recent of the the association between 24-h activity rhythms and neurodegenerative disorders.

6. Van Someren EJ, Riemersma-Van Der Lek RF. Live to the rhythm, slave to the rhythm. Sleep Med Rev. 2007;11(6):465-84.

7. Logan RW, McClung CA. Rhythms of life: circadian disruption and brain disorders across the lifespan. Nat Rev Neurosci. 2019;20(1): 49-65. Recent review on aging and 24-h activity rhythms.

8. Licher S, Heshmatollah A, van der Willik KD, Stricker BHC, Ruiter R, de Roos EW, et al. Lifetime risk and multimorbidity of non-communicable diseases and disease-free life expectancy in the general population: a population-based cohort study. PLoS Med. 2019;16(2):e1002741.

9. Tranah GJ, Blackwell T, Ancoli-Israel S, Paudel ML, Ensrud KE, Cauley JA, et al. Circadian activity rhythms and mortality: the study of osteoporotic fractures. J Am Geriatr Soc. 2010;58(2):282-91.

10. Depner CM, Cheng PC, Devine JK, Khosla S, de Zambotti M, Robillard R, et al. Wearable technologies for developing sleep and circadian biomarkers: a summary of workshop discussions. Sleep. 2019;43(2):zsz254.

11.• Marler MR, Gehrman P, Martin JL, Ancoli-Israel S. The sigmoidally transformed cosine curve: a mathematical model for circadian rhythms with symmetric non-sinusoidal shapes. Stat Med. 2006;25(22):3893-904. Key method paper for cosinor analyses of 24-h activity rhythms.

12.• Van Someren EJW. Actigraphic monitoring of movement and restactivity rhythms in aging, Alzheimer's disease, and Parkinson's disease. IEEE Trans Rehabil Eng. 1997;5(4):394-8. Key method paper for non-parametric analyses of 24-h activity rhythms.

13. Mitchell JA, Quante M, Godbole S, James P, Hipp JA, Marinac CR, et al. Variation in actigraphy-estimated rest-activity patterns by demographic factors. Chronobiol Int. 2017;34(8):1042-56.

14. Smagula S, Gujral S, Capps C, Krafty R. A systematic review of evidence for a role of rest-activity rhythms in dementia. Front Psych. 2019;10:778.

15. Rogers-Soeder TS, Blackwell T, Yaffe K, Ancoli-Israel S, Redline $\mathrm{S}$, Cauley JA, et al. Rest-activity rhythms and cognitive decline in older men: the osteoporotic fractures in men sleep study. J Am Geriatr Soc. 2018;66(11):2136-43. One of the few large-scale, longitudinal, population-based studies investigating 24-h activity rhythms and neurodegenerative disorders.

16. Musiek ES, Bhimasani M, Zangrilli MA, Morris JC, Holtzman DM, Ju Y-ES. Circadian rest-activity pattern changes in aging and preclinical Alzheimer disease. JAMA Neurol. 2018;75(5):582-90.

17. Hori H, Koga N, Hidese S, Nagashima A, Kim Y, Higuchi T, et al. 24-h activity rhythm and sleep in depressed outpatients. J Psychiatr Res. 2016;77:27-34.

18. Wennman H, Pietilä A, Rissanen H, Valkeinen H, Partonen T, Mäki-Opas T, et al. Gender, age and socioeconomic variation in 24-hour physical activity by wrist-worn accelerometers: the FinHealth 2017 Survey. Sci Rep. 2019;9(1):6534.

19. Ramirez V, Shokri-Kojori E, Cabrera EA, Wiers CE, Merikangas $\mathrm{K}$, Tomasi D, et al. Physical activity measured with wrist and ankle accelerometers: age, gender, and BMI effects. PloS One. 2018;13(4):e0195996.

20. Luik AI, Zuurbier LA, Hofman A, Van Someren EJW, Tiemeier H Stability and fragmentation of the activity rhythm across the sleepwake cycle: the importance of age, lifestyle, and mental health. Chronobiol Int. 2013;30(10):1223-30.

21. Lyall LM, Wyse CA, Graham N, Ferguson A, Lyall DM, Cullen B, et al. Association of disrupted circadian rhythmicity with mood disorders, subjective wellbeing, and cognitive function: a crosssectional study of 91105 participants from the UK Biobank. Lancet Psychiatry. 2018;5(6):507-14. One of the few large-scale, population-based studies investigating 24-h activity rhythms and mood.

22. Weldemichael DA, Grossberg GT. Circadian rhythm disturbances in patients with Alzheimer's disease: a review. Int J Alzheimers Dis. 2010;2010:1-9.

23. Hatfield CF, Herbert J, van Someren EJ, Hodges JR, Hastings MH. Disrupted daily activity/rest cycles in relation to daily cortisol rhythms of home-dwelling patients with early Alzheimer's dementia. Brain. 2004;127(Pt 5):1061-74.

24. Wang JL, Lim AS, Chiang WY, Hsieh WH, Lo MT, Schneider JA, et al. Suprachiasmatic neuron numbers and rest-activity circadian rhythms in older humans. Ann Neurol. 2015;78(2):317-22.

25. Collaborators GBDD. Global, regional, and national burden of Alzheimer's disease and other dementias, 1990-2016: a systematic analysis for the global burden of disease study 2016. Lancet Neurol. 2019;18(1):88-106.

26. Witting W, Kwa IH, Eikelenboom P, Mirmiran M, Swaab DF. Alterations in the circadian rest-activity rhythm in aging and Alzheimer's disease. Biol Psychiatry. 1990;27(6):563-72.

27. Videnovic A, Lazar AS, Barker RA, Overeem S. 'The clocks that time us' - circadian rhythms in neurodegenerative disorders. Nat Rev Neurol. 2014;10(12):683.

28. Weissova K, Bartoš A, Sládek M, Novakova M, Sumová A. Moderate changes in the circadian system of Alzheimer's disease patients detected in their home environment. PLoS One. 2016;11(1):e0146200.

29. Van Someren EJW, Oosterman JM, Van Harten B, Vogels RL, Gouw AA, Weinstein HC, et al. Medial temporal lobe atrophy relates more strongly to sleep-wake rhythm fragmentation than to age or any other known risk. Neurobiol Learn Mem. 2019;160: $132-8$

30. Wams EJ, Wilcock GK, Foster RG, Wulff K. Sleep-wake patterns and cognition of older adults with amnestic mild cognitive impairment (aMCI): a comparison with cognitively healthy adults and moderate Alzheimer's disease patients. Curr Alzheimer Res. 2017;14(10):1030-41.

31. Saito Y, Kume Y, Kodama A, Sato K, Yasuba M. The association between circadian rest-activity patterns and the behavioral and psychological symptoms depending on the cognitive status in Japanese nursing-home residents. Chronobiol Int. 2018;35(12):1670-9. 
32. Hooghiemstra AM, Eggermont LHP, Scheltens P, van der Flier WM, Scherder EJA. The rest-activity rhythm and physical activity in early-onset dementia. Alzheimer Dis Assoc Disord. 2015;29(1): 45-9.

33. Bromundt V, Wirz-Justice A, Boutellier M, Winter S, Haberstroh $\mathrm{M}$, Terman M, et al. Effects of a dawn-dusk simulation on circadian rest-activity cycles, sleep, mood and well-being in dementia patients. Exp Gerontol. 2019;124:110641. Experimental study providing new insight in interventions targeting 24-h acitivity rhythms in patients with dementia.

34. Smagula SF, Krafty RT, Taylor BJ, Martire LM, Schulz R, Hall MH. Rest-activity rhythm and sleep characteristics associated with depression symptom severity in strained dementia caregivers. J Sleep Res. 2017;26(6):718-25.

35. Manousakis JE, Scovelle AJ, Rajaratnam SMW, Naismith SL, Anderson C. Advanced circadian timing and sleep fragmentation differentially impact on memory complaint subtype in subjective cognitive decline. J Alzheimers Dis. 2018;66(2):565-77.

36. Ortiz-Tudela E, Martinez-Nicolas A, Díaz-Mardomingo C, GarcíaHerranz S, Pereda-Pérez I, Valencia A, et al. The characterization of biological rhythms in mild cognitive impairment. Biomed Res Int. 2014;2014:524971.

37. Zeitzer JM, Blackwell T, Hoffman AR, Cummings S, Ancoli-Israel $\mathrm{S}$, Stone K, et al. Daily patterns of accelerometer activity predict changes in sleep, cognition, and mortality in older men. J Geronotol A Biol Sci Med Sci. 2017;73(5):682-7.

38. Tranah GJ, Blackwell T, Stone KL, Ancoli-Israel S, Paudel ML, Ensrud KE, et al. Circadian activity rhythms and risk of incident dementia and mild cognitive impairment in older women. Ann Neurol. 2011;70(5):722-32.

39. Smagula SF, Chahine L, Metti A, Rangarajan A, Aizenstein HJ, Tian $\mathrm{Q}$, et al. Regional gray matter volume links rest-activity rhythm fragmentation with past cognitive decline. Am J Geriatr Psychiatry. 2019;28(2):248-51. Study relating cognitive decline measured over 14 years with 24-h activity rhythms.

40. Videnovic A, Willis GL. Circadian system - a novel diagnostic and therapeutic target in Parkinson's disease? Mov Disord. 2016;31(3): 260-9.

41. La Morgia C, Ross-Cisneros FN, Sadun AA, Carelli V. Retinal ganglion cells and circadian rhythms in Alzheimer's disease, Parkinson's disease, and beyond. Front Neurol. 2017;8:162.

42. Wu JQ, Li P, Stavitsky Gilbert K, Hu K, Cronin-Golomb A. Circadian rest-activity rhythms predict cognitive function in early Parkinson's disease independently of sleep. Mov Disord Clin Pract. 2018;5(6):614-9.

43. Leng Y, Goldman SM, Cawthon PM, Stone KL, Ancoli-Israel S, Yaffe K. Excessive daytime sleepiness, objective napping and 11year risk of Parkinson's disease in older men. Int J Epidemiol. 2018;47(5):1679-86.

44. Seifalian A, Hart A. Circadian rhythms: will it revolutionise the management of diseases? J Lifestyle Med. 2019;9(1):1. Overview of potential of circadian rhythm interventions for improving health.

45. Ferguson A, Lyall LM, Ward J, Strawbridge RJ, Cullen B, Graham $\mathrm{N}$, et al. Genome-wide association study of circadian rhythmicity in 71,500 UK Biobank participants and polygenic association with mood instability. EBioMedicine. 2018;35:279-87. Large genome-wide association study of 24-h activity rhythms.

46. American Psychiatric Association. Diagnostic and statistical manual of mental disorders; fifth edition. Arlington: American Psychiatric Association; 2013.

47. World Health O. Depression and other common mental disorders: global health estimates. 2017.

48. Difrancesco S, Lamers F, Riese H, Merikangas KR, Beekman ATF, van Hemert AM, et al. Sleep, circadian rhythm, and physical activity patterns in depressive and anxiety disorders: a 2-week ambulatory assessment study. Depress Anxiety. 2019;36(10):97586. Assessment of 24-h activity rhythms in patients currently suffering from depression/anxiety, remitted depression/anxiety or no history of depression/anxiety.

49. Smagula SF. Opportunities for clinical applications of rest-activity rhythms in detecting and preventing mood disorders. Curr Opin Psychiatry. 2016;29(6):389.

50. Kelly RM, Healy U, Sreenan S, McDermott JH, Coogan AN. Clocks in the clinic: circadian rhythms in health and disease. Postgrad Med J. 2018;94(1117):653-8.

51. Vadnie CA, McClung CA. Circadian rhythm disturbances in mood disorders: insights into the role of the suprachiasmatic nucleus. Neural Plast. 2017;2017:1504507.

52. Luik AI, Zuurbier LA, Direk N, Hofman A, Van Someren EJW, Tiemeier H. 24-hour activity rhythm and sleep disturbances in depression and anxiety: a population-based study of middle-aged and older persons. Depress Anxiety. 2015;32(9):684-92.

53. $\mathrm{Au} \mathrm{J}$, Reece J. The relationship between chronotype and depressive symptoms: a meta-analysis. J Affect Disord. 2017;218:93-104.

54. Antypa N, Verkuil B, Molendijk M, Schoevers R, Penninx BWJH, Van Der Does W. Associations between chronotypes and psychological vulnerability factors of depression. Chronobiol Int. 2017;34(8):1125-35.

55. White KH, Rumble ME, Benca RM. Sex differences in the relationship between depressive symptoms and actigraphic assessments of sleep and rest-activity rhythms in a population-based sample. Psychosom Med. 2017;79(4):479.

56. Smagula SF, Boudreau RM, Stone K, Reynolds Iii CF, Bromberger JT, Ancoli-Israel S, et al. Latent activity rhythm disturbance subgroups and longitudinal change in depression symptoms among older men. Chronobiol Int. 2015;32(10):1427-37.

57. Wichniak A, Wierzbicka A, Walęcka M, Jernajczyk W. Effects of antidepressants on sleep. Curr Psychiatry Rep. 2017;19(9):63.

58. McGlashan EM, Drummond SPA, Cain SW. Evening types demonstrate reduced SSRI treatment efficacy. Chronobiol Int. 2018;35(8):1175-8.

59. Swanson LM, Burgess HJ, Huntley ED, Bertram H, Mooney A, Zollars J, et al. Relationships between circadian measures, depression, and response to antidepressant treatment: a preliminary investigation. Psychiatry Res. 2017;252:262-9. Experimental study assessing the possible role of 24 -h activity rhythms in antidpressant treatment.

60. Duncan WC Jr, Slonena E, Hejazi NS, Brutsche N, Kevin CY, Park $\mathrm{L}$, et al. Motor-activity markers of circadian timekeeping are related to ketamine's rapid antidepressant properties. Biol Psychiatry. 2017;82(5):361-9.

61. Melo MCA, Abreu RLC, Neto VBL, de Bruin PFC, de Bruin VMS. Chronotype and circadian rhythm in bipolar disorder: a systematic review. Sleep Med Rev. 2017;34:46-58.

62. Smagula SF, Krafty RT, Thayer JF, Buysse DJ, Hall MH. Restactivity rhythm profiles associated with manic-hypomanic and depressive symptoms. J Psychiatr Res. 2018;102:238-44.

63. Rumble ME, Dickson D, McCall WV, Krystal AD, Case D, Rosenquist PB, et al. The relationship of person-specific eveningness chronotype, greater seasonality, and less rhythmicity to suicidal behavior: a literature review. J Affect Disord. 2018;227: $721-30$.

64. Benard V, Etain B, Vaiva G, Boudebesse C, Yeim S, Benizri C, et al. Sleep and circadian rhythms as possible trait markers of suicide attempt in bipolar disorders: an actigraphy study. J Affect Disord. 2019;244:1-8.

65. Wee ZY, Yong SWL, Chew QH, Guan C, Lee TS, Sim K. Actigraphy studies and clinical and biobehavioural correlates in schizophrenia: a systematic review. J Neural Transm. 2019;126(5):531-58. 
66. Bromundt V, Köster M, Georgiev-Kill A, Opwis K, Wirz-Justice A, Stoppe G, et al. Sleep-wake cycles and cognitive functioning in schizophrenia. Br J Psychiatry. 2011;198(4):269-76.

67. Papandreou C, Díaz-López A, Babio N, Martínez-González MA, Bulló M, Corella $\mathrm{D}$, et al. Long daytime napping is associated with increased adiposity and type 2 diabetes in an elderly population with metabolic syndrome. J Clin Med. 2019;8(7):1053.

68. Feliciano EMC, Quante M, Weng J, Mitchell JA, James P, Marinac $\mathrm{CR}$, et al. Actigraphy-derived daily rest-activity patterns and body mass index in community-dwelling adults. Sleep. 2017;40(12): zsx168.

69. Sohail S, Yu L, Bennett DA, Buchman AS, Lim ASP. Irregular 24hour activity rhythms and the metabolic syndrome in older adults. Chronobiol Int. 2015;32(6):802-13.

70. Paudel ML, Taylor BC, Ancoli-Israel S, Stone KL, Tranah G, Redline S, et al. Rest/activity rhythms and cardiovascular disease in older men. Chronobiol Int. 2011;28(3):258-66. One of the few large-scale, longitudinal, population-based studies investigating 24-h activity rhythms and cardiovascular disease.

71. Havakuk O, Zukerman N, Flint N, Sadeh B, Margolis G, Konigstein M, et al. Shift work and the risk of coronary artery disease: a cardiac computed tomography angiography study. Cardiology. 2018;139(1):11-6.

72. Vetter C, Dashti HS, Lane JM, Anderson SG, Schernhammer ES, Rutter MK, et al. Night shift work, genetic risk, and type 2 diabetes in the UK biobank. Diabetes Care. 2018;41(4):762-9.

73. Morris CJ, Purvis TE, Hu K, Scheer FAJL. Circadian misalignment increases cardiovascular disease risk factors in humans. Proc Natl Acad Sci. 2016;113(10):E1402-E11.

74. Buxton OM, Cain SW, O'Connor SP, Porter JH, Duffy JF, Wang W, et al. Adverse metabolic consequences in humans of prolonged sleep restriction combined with circadian disruption. Sci Transl Med. 2012;4(129):129ra43-ra43.

75. Bernatchez MS, Savard J, Ivers H. Disruptions in sleep-wake cycles in community-dwelling cancer patients receiving palliative care and their correlates. Chronobiol Int. 2018;35(1):49-62.

76. Chang W-P, Smith R, Lin C-C. Age and rest-activity rhythm as predictors of survival in patients with newly diagnosed lung cancer.
Chronobiol Int. 2018;35(2):188-97. Assessment of the possible role of 24-h activity rhythms in survival of cancer patients.

77. Kuo L-C, Chang W-P, Huang H-C, Lin C-C. Association of timevarying rest-activity rhythm with survival in older adults with lung cancer. Cancer Nurs. 2019;43(1):45-51.

78. Cash E, Duck CR, Brinkman C, Rebholz W, Albert C, Worthen M, et al. Depressive symptoms and actigraphy-measured circadian disruption predict head and neck cancer survival. Psycho-oncology. 2018;27(10):2500-7.

79. Sultan A, Choudhary V, Parganiha A. Worsening of rest-activity circadian rhythm and quality of life in female breast cancer patients along progression of chemotherapy cycles. Chronobiol Int. 2017;34(5):609-23.

80. Mitolo M, Tonon C, La Morgia C, Testa C, Carelli V, Lodi R. Effects of light treatment on sleep, cognition, mood, and behavior in Alzheimer's disease: a systematic review. Dement Geriatr Cogn Disord. 2018;46(5-6):371-84.

81. Fifel K, Videnovic A. Chronotherapies for Parkinson's disease. Prog Neurobiol. 2019;174:16-27.

82. Leggett AN, Conroy DA, Blow FC, Kales HC. Bright light as a preventive intervention for depression in late-life: a pilot study on feasibility, acceptability, and symptom improvement. Am J Geriatr Psychiatry. 2018;26(5):598-602.

83. Boivin DB, James FO. Light treatment and circadian adaptation to shift work. Ind Health. 2005;43(1):34-48.

84. Johnson JA, Garland SN, Carlson LE, Savard J, Simpson JSA, Ancoli-Israel S, et al. Bright light therapy improves cancer-related fatigue in cancer survivors: a randomized controlled trial. J Cancer Surviv. 2018;12(2):206-15.

85. Hopkins S, Lloyd Morgan P, Jm Schlangen L, Williams P, Skene DJ, Middleton B. Blue-enriched lighting for older people living in care homes: effect on activity, actigraphic sleep, mood and alertness. Curr Alzheimer Res. 2017;14(10):1053-62.

Publisher's Note Springer Nature remains neutral with regard to jurisdictional claims in published maps and institutional affiliations. 